\title{
UNIVERSITYOF
}

FORWARD

THINKING

WESTMINSTER用

WestminsterResearch

http://www.westminster.ac.uk/westminsterresearch

\section{Turmeric (Curcuma longa L.) products: What quality differences exist?}

Chatzinasiou, L., Booker, A., MacLennan, E., Mackonochie, M. and Heinrich, $M$.

NOTICE: this is the authors' version of a work that was accepted for publication in Journal of Herbal Medicine. Changes resulting from the publishing process, such as peer review, editing, corrections, structural formatting, and other quality control mechanisms may not be reflected in this document. Changes may have been made to this work since it was submitted for publication. A definitive version was subsequently published in Journal of Herbal Medicine, DOI: 10.1016/j.hermed.2019.100281, 2019.

The final definitive version in Journal of Herbal Medicine is available online at:

https://dx.doi.org/10.1016/j.hermed.2019.100281

(C) 2019. This manuscript version is made available under the CC-BY-NC-ND 4.0 license https://creativecommons.org/licenses/by-nc-nd/4.0/

The WestminsterResearch online digital archive at the University of Westminster aims to make the research output of the University available to a wider audience. Copyright and Moral Rights remain with the authors and/or copyright owners.

Whilst further distribution of specific materials from within this archive is forbidden, you may freely distribute the URL of WestminsterResearch: ((http://westminsterresearch.wmin.ac.uk/)).

In case of abuse or copyright appearing without permission e-mail repository@westminster.ac.uk 
Turmeric (Curcuma longa L.) products: What quality differences exist?

\begin{abstract}
Background: Curcuma longa L. (root and rhizome), commonly known as turmeric, is a plant of high medicinal and economic value globally, including within the EU where it is mainly used as a food and food supplement. Its key active ingredients have been studied extensively, but concerns remain about the composition and thus the quality of extracts and products on the market. Recently, problems of adulteration with other Curcuma species and general poor quality have been detected within crude turmeric powders and final products. This study aimed to investigate the chemical variation of turmeric finished products available in the UK and identify the quality of turmeric products obtained from specific stages of the supply chain, with a focus on quality differences in the UK. ${ }^{1} \mathrm{H}-\mathrm{NMR}$ spectroscopy metabolomics coupled to Principle Component Analysis (PCA) and HPTLC methods were used for the phytochemical and metabolomics investigation of 72 turmeric samples, including crude powders, intermediate products from different manufacturing stages and commercial products obtained from health food stores and the Internet. A significant variation in the chemical composition of turmeric finished products, especially in the ones containing turmeric extracts, was found. Several products were found to contain only curcumin or curcuminoids, while the other active ingredients and marker compounds of $C$. longa were lacking. Products obtained from different stages of the Pukka value chain presented similar chemical composition, with the intermediate products (S57-59) showing the highest amount of active ingredients. Consequently, the manufacturing practices involved in the production of turmeric food supplements are critical stages to obtain high-quality final products. Often the focus has been only on the primary material, its natural variation and forms of production. This study highlights the importance of developing well-controlled integrated systems for the quality assurance of dietary supplements throughout the supply chain from seed to final product.
\end{abstract}

Keywords

Quality control, value chains, Curcuma longa, Turmeric, Food supplements

\title{
Abbreviations
}

HPTLC-High performance thin layer chromatography, NMR-Nuclear Magnetic Resonance, FS/P-Food Supplement/Powder, FS/E-Food Supplement/Extract, FS/P+EFood Supplement/Powder+Extract, TP/PUKKA-Turmeric Powder/PUKKA, TWE/PUKKA-Turmeric Wholistic Extract/PUKKA, TP/DS-Turmeric Powder/ Different Suppliers, CS-Curcumin Standard, PS-Piperine Standard, TS-Turmerone Standard, FP-final product, PTP-Pukka turmeric powder 



\section{Introduction}

Curcuma longa L. (Zingiberaceae) root and rhizome, commonly known as turmeric, has a long history of medicinal and nutritional applications, especially in Asian countries. Over the past few years, its importance has been widely recognised globally, and, therefore, numerous studies have been published (Prasad and Aggarwal, 2011).

A large variety of turmeric products are readily accessible via the internet or health food shops, mainly as food supplements, and these vary in formulation, concentration of supposed active substances, additional ingredients or recommended dosages.

Successive studies have highlighted the inconsistent quality of Curcuma longa and Curcuma supplements as well as the variability of the active metabolites (Dixit et al., 2009; Li et al., 2011; Starling, 2009). Booker et al (2014a) reported a wide range of curcuminoid content within UK sourced samples and a lack of important compounds typically found within the volatile oils, e.g. ar-turmerone (Booker et al., 2014a). This is partly due to a lack of consensus on the relative importance of different constituents relevant for its therapeutic benefits, and partly due to differing quality within the various markets.

Although curcumin presents one of the most pharmacologically important constituents in turmeric, it is claimed that the low bioavailability restricts its clinical applications (Shishu, 2010). Several studies have shown that curcumin is barely absorbed by the gastrointestinal tract when ingested, due to rapid metabolic breakdown by the liver (Ammon et al., 1992; Anand et al., 2009; Li et al., 2011; Ravindranath and Chanfrasekhara, 1981; Wang et al., 1997).

A common approach to attempt to enhance the oral bioavailability of curcumin is its combination with other natural compounds, such as piperine and ginger (Maharao et al., 2016). Piperine, the main active compound found in black and long peppers (Pipper nigrum L. and Piper longum L.), has been reported to inhibit the glucuronidation of many drugs, as well as improving curcumin bioavailability (Prasad et al., 2014). Therefore, a number of finished products on the market now contain piperine, or black or long pepper in attempt to make these products more attractive to consumers.

However, other constituents in turmeric, such as the compounds found in the essential oils, and metabolites such as tetrahydro-curcuminoids, may play an important role, and there is increasing evidence to suggest a local effect of turmeric on the microbiome and local inflammation levels in the gut (Kukula-Koch et al., 2018; Santosh et al., 2017). Toden et al (2017) found that a combination of curcumin and turmeric essential oils had superior anti-inflammatory activity to curcumin alone in a mouse model of colitis. Additionally, a pilot human study of oral bioavailability of a range of turmeric formulations found that a combination of curcumin with essential oils had superior bioavailability to both curcumin alone and a combination of curcumin and piperine (Antony et al, 2008). Other studies, however, have found only minimal increases in relative absorption of curcuminoids with the addition of essential oils (Purpura et al, 2017).

As further research is needs to determine the relative bioavailability and therapeutic benefits of different turmeric-derived formulations, it will be useful to have tools able 
to group preparations according to their active ingredients to compare with therapeutic benefits.

In essence, this results in two distinct but related challenges:

- The need to understand the composition of the turmeric materials which are commercialised

- The development of optimum preparations

\section{Aims and objectives}

In this paper we focus on the first of these two tasks. Therefore, the main scientific aims of this study were:

- To understand the spectrum of bioactive constituents found within Pukka products and their concentration within the product using an approach combining NMRmetabolomic analysis and HPTLC.

- To understand the supply chain by investigating the compositional differences among a range of dried powder and fresh turmeric powdered rhizome from India.

- To examine the compositional diversity among finished turmeric products available in the UK.

- To identify any adulterants within these products.

\section{Materials and methods}

\section{Samples collection}

72 samples were analysed in total. 50 finished turmeric products were purchased from stores mainly in Central London, but also Germany, the USA and from the Internet. The products were formulated in capsules (43), tablets (2), softgels (2), powder (1) and extracts (2) and they claimed to contain either plant extract or crude ground material of the plant or a combination of plant extract and crude ground material. 22 samples were provided by Pukka Herbs, including Wholistic Turmeric ${ }^{\circledR}$ products (6; S41-46), crude ground material from India (10), Pukka Turmeric Wholistic Extract (3; S57-59) and Pukka Turmeric Powder (3). The finished products were formulated into capsules and claimed to contain both plant extract and crude ground material of the plant.

\section{Reference standards}

Curcumin reference standard (mixture of three curcuminoids, Lot 69H3457) was obtained from Sigma-Aldrich, (S)-ar-Turmerone from LKT Laboratories (Lot 2598838) and piperine standard form LKT Laboratories (Lot 2598684).

\section{${ }^{1}$ H-NMR Spectroscopy}

${ }^{1} \mathrm{H}-\mathrm{NMR}$ spectra of turmeric samples was measured on a Bruker Avance $500 \mathrm{MHz}$ spectrophotometer (Bruker Analytik Rheinstetten, Germany) equipped with a $5 \mathrm{~mm}$ cryoprobe head and operating at proton frequency of $500.13 \mathrm{MHz}$ at temperature 298 $\mathrm{K}$. The acquisition parameters were: $0.16 \mathrm{~Hz} /$ point, pulse width $=30^{\circ}$ and relaxation delay $=1 \mathrm{~s}, 256$ scans. TOPSPIN software version 3.2 was used for spectra acquisition 
and processing. Centrifuge (Eppendorf $^{\mathrm{TM}}$ MiniSpin $^{\mathrm{TM}}$, 1010813), ultrasonic bath $\left(\mathrm{VWR}^{\mathrm{TM}}, 110233\right)$ and analytical balance (METTLER TOLEDO, AB135-S/FACT) were also used for the sample preparation.

Sample preparation for ${ }^{1} \mathrm{H}-\mathrm{NMR}$ analysis

The method for the analysis of turmeric samples was based on a method used by Booker et al (2014a). DMSO-d6 was selected as extracting solvent, due to the increased amount of peaks present in ${ }^{1} \mathrm{H}-\mathrm{NMR}$ spectra in a previous study (Booker et al., 2014a). Approximately $20 \mathrm{mg}$ of all the samples were weighted into a $1.5 \mathrm{ml}$ reaction tube and $1.0 \mathrm{ml}$ of deuterated DMSO-d6 containing 0.05\% TMS was added.

The mixtures were shaken on a rotary mixer for $60 \mathrm{~s}$, sonicated for $15 \mathrm{~min}$ at room temperature, and allowed to stand for $1 \mathrm{~h}$. Then, the mixtures were centrifuged for 10 min at $14,000 \mathrm{rpm}$ and $600 \mu \mathrm{l}$ of the supernatant was transferred to a $5 \mathrm{~mm}$ diameter NMR spectroscopy tube. The samples were submitted for NMR analysis.

\section{Principle Component Analysis (PCA)}

TOPSPIN software version 3.2 was used to acquire and process the spectra. The spectra were acquired with 256 scans, requiring about $18 \mathrm{~min}$ acquisition time. The scans were locked at zero on the TMS peak. The residual solvent signal for DMSO was excluded. The samples, including commercial products, crude materials and standards, were numbered 1 to 75. Microsoft EXCEL was used for the analysis of the data. Sample 42 was analysed twice (421, duplicate sample) as a control in order to check the robustness and reproducibility of both PCA and NMR. The data were imported to SIMCA version 14.0 software for PCA analysis.

\section{HPTLC}

The CAMAG HPTLC equipment was used, composed of Visualizer, Automatic TLC sampler 4 (ATS4) and TLC sampler LINOMAT 5, Automatic Developing Chamber 2 (ADC2), Chromatogram Immersion Device III for derivatisation, TLC plate heater III and TLC scanner coupled to VisionCats version 1.4 software. Centrifuge (Eppendorf ${ }^{\mathrm{TM}}$ MiniSpin $^{\mathrm{TM}}$, 1010813), ultrasonic bath $\left(\mathrm{VWR}^{\mathrm{TM}}\right.$, 110233) and analytical balance (METTLER TOLEDO, AB135-S/FACT) were also used for the sample preparation.

Sample preparation for HPTLC

The method for the analysis of turmeric samples was based on a method used by (Booker et al., 2014b). The method for the extraction of the samples was based on a standardized method used at CAMAG laboratories and Booker et al (2014a). Methanol was used for the determination of curcuminoids and toluene for the determination of essential oils. Two different developing systems were applied, one for curcuminoid determination (Toluene: acetic acid, 4:1) and another one for essential oils (Dichloromethane). About $200 \mathrm{mg}$ of all samples were weighted into $10 \mathrm{ml}$ reaction tubes and $4.0 \mathrm{ml}$ of solvent was added. Two different solvents were tested: methanol for curcuminoid and dye adulterant determination and toluene for the determination of 
essential oils. The mixture was mixed using a rotary mixer for $60 \mathrm{~s}$ and sonicated for $10 \mathrm{~min}$. Then, the supernatant solution was transferred to a vial and was centrifuged for $10 \mathrm{~min}$ at $14,000 \mathrm{rpm}$. The samples were submitted for HPTLC analysis.

\section{HPTLC Analysis}

HPTLC glass 20x10 cm, Si 60 F254 plates were loaded with the turmeric samples in the CAMAG Automatic TLC Sampler 4. Then $4 \mu$ of each sample and standard were applied to the plate as $8 \mathrm{~mm}$ bands. The maximum number of samples per plate was 15 .

The plates were obtained from CAMAG Laboratories, Muttenz, Switzerland and developed using a CAMAG Automatic Development Chamber ADC 2 using two different mobile phases, toluene: acetic acid (4:1) for curcuminoid determination and dichloromethane for the determination of essential oils. The lab temperature was $23^{\circ} \mathrm{C}$. The humidity of the tank was controlled at about 33\%, after the treatment with magnesium chloride solution. After the development the plate was dried for $5 \mathrm{~min}$ and then heated at $100^{\circ} \mathrm{C}$ for $2 \mathrm{~min}$, using a CAMAG automatic TLC heater. The plates were derivatised by dipping in anisaldehyde reagent for the detection of both curcuminoids and essential oils, using a CAMAG chromatogram immersion device III and heated to $100^{\circ} \mathrm{C}$ for $3 \mathrm{~min}$ on a plate heater. The plates were inspected using a CAMAG visualizer under white light and at UV $254 \mathrm{~nm}$, after the development. After the derivatisation, the plates were visualized under white light and UV $366 \mathrm{~nm}$. The results were uploaded to the VisionCats version 1.4 software.

\section{Results and discussion}

\section{${ }^{1} \mathrm{H}-\mathrm{NMR}$ spectroscopy coupled to PCA-metabolomics investigation Finished products}

${ }^{1} \mathrm{H}-\mathrm{NMR}$ spectroscopy coupled to Principal Component Analysis (PCA) was used to investigate the variation in the chemical composition of turmeric products within different stages of the value chain. Samples (numbered as S1-S72) were crude materials from different suppliers (S63-72), processed turmeric powders (S60-62, manufacturing stage), extracted materials (S57-59, manufacturing stage) and finished commercial products (S1-56). Visual inspection of the acquired NMR spectra shows that the variation between the samples is significant. Several samples presented similar spectra, while others showed almost undetectable quantities of Curcuma longa $\mathrm{L}$. In order to compare the individual ${ }^{1} \mathrm{H}-\mathrm{NMR}$ spectra, curcumin, piperine and ar-turmerone standards were also analysed.

The samples were divided into groups, depending on their origin, use and form/preparation and were separated using PCA scores plot. Two different plots were created, one to compare the different turmeric finished products and one to investigate products obtained from four different stages of the Pukka value chain. In order to better understand the differences and similarities among turmeric samples, the region associated with the most characteristic peaks for curcuminoids, ar-turmerone and piperine (6-8 $\mathrm{ppm}$ ) was selected for observation. 


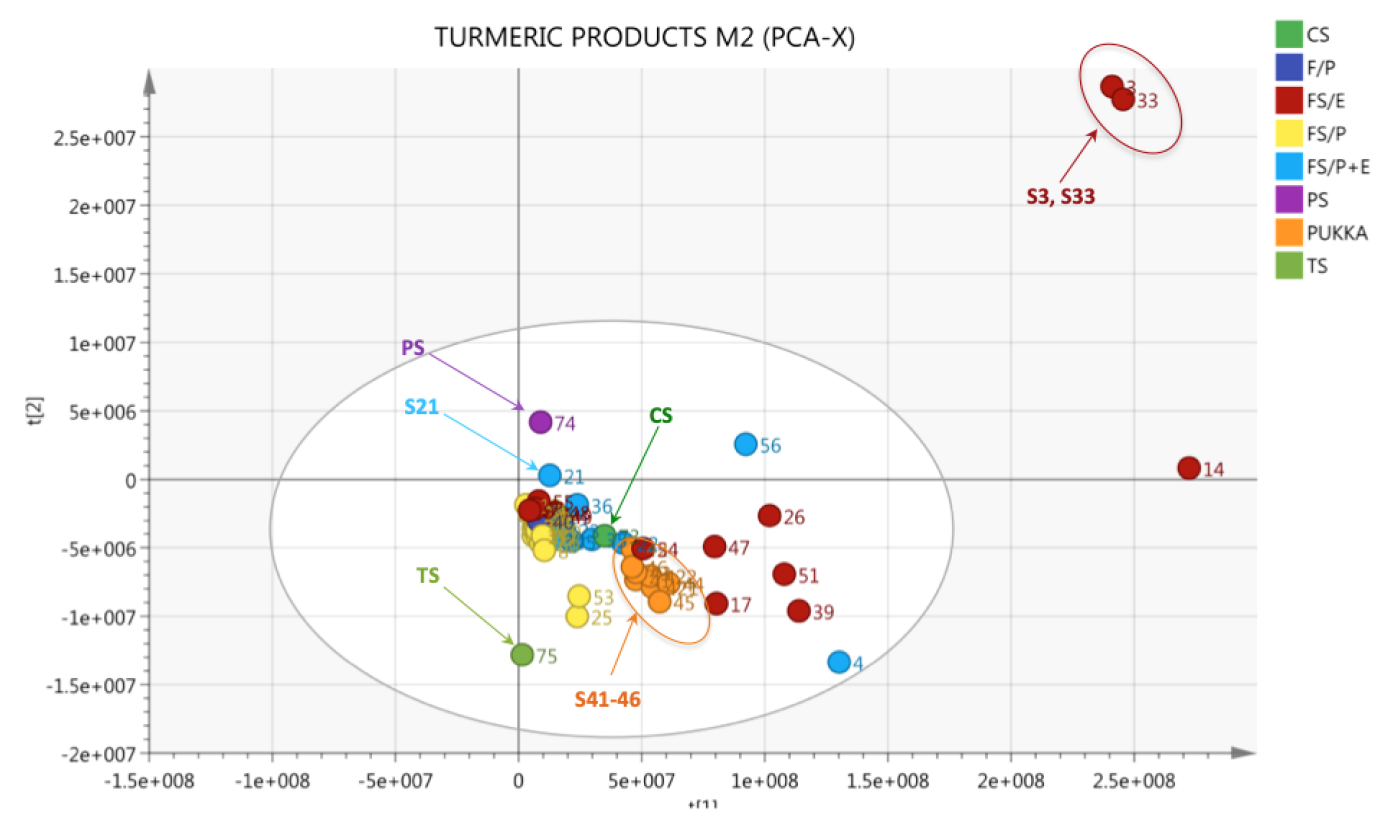

Figure 1: Scores plot in the chemical shift region 6-8 ppm, comparing different turmeric finished products containing powdered material, extract or a combination of both. Food Supplement/Powder (FS/P), Food Supplement/ Extract (FS/E), Food Supplement/ Powder+ Extract (FS/P+E). Curcumin, piperine and ar-turmerone standards are also presented (CS, PS, TS). X axis: Principle Component 1 (PC1), Y axis: Principle Component 2 (PC2). (n=60)

The products composed only of turmeric powder were grouped together (yellow), while turmeric extracts showed important metabolite variability and no distinct clustering occurred (red) (Fig 1). Several of them were placed out of the score plot, indicating possible absence of $C$. longa constituents or adulteration with other Curcuma species. The significant variation in the distribution of turmeric extracts could be also attributed to the different extracting solvents used to formulate each product. However, the information related to their extraction process was not provided on the label, and, therefore, they could not be separated to smaller clusters. These results imply that the processing of the crude material is a significant but, in the herbal industries and by the general public, often ignored complex series of stages within the production of turmeric food supplements.

Finished products S41-46 appeared to be close to ar-turmerone and curcumin standards (S75 and S73 respectively), which shows that these samples were likely to be high in these constituents. Subsequent quantitative analysis confirmed that $S 41=46$ were high in ar-turmerone, while their curcumin concentration was average (Fig $2 \& 3$ ).Sample 21 , containing the highest amount of piperine ( $20 \mathrm{mg}$ according to the label), appeared very close to the piperine standard (S74), as expected (Fig 1). Samples 3, 33 and 14 (extracts) appeared to be out of the scores plot, which indicates that they significantly differ from the other samples in the chemical shift region 6-8 ppm. Samples 3 and 33 were very well grouped, signifying that they possibly had similar metabolic profiles. Subsequent HPTLC analysis showed that they were strong in curcumin and piperine, but did not contain any other active ingredient from $C$. longa, while sample 14 contained only the three major curcuminoids and piperine.

The quantitative evaluation of the samples was based on the average peak intensity they presented in three specific chemical shift regions associated with ar-turmerone (7.04- 
$7.13 \mathrm{ppm}$ ), curcuminoids (6.73- $6.84 \mathrm{ppm})$ and piperine (6.851- $7.0 \mathrm{ppm})$. The samples were sorted in descending order, according to their average absorbance values in these regions, and, therefore, their concentration in ar-turmerone and piperine could be determined. The higher the peak intensity is in a specific region, the higher is the concentration of a specific compound or a group of compounds in the sample.

Ar-turmerone is an important active constituent of turmeric essential oil, which provides $C$. longa with its unique fragrance and has been found to increase the uptake of curcumin by human intestinal epithelial Caco-2 cells in vitro (Park et al., 2012). A combination of both sesquiterpenoids and curcuminoids had superior pharmacological effects in a mouse model of diabetes to extracts of either compound type alone (Nishiyama et al, 2005). Independently of curcumin, ar-turmerone has been found to have tumour inhibiting and anti-inflammatory activity (Aggarwal et al., 2013). This compound can only be detected in $C$. longa, while it is absent in $C$. aromatica and $C$. zanthorrhiza (Booker, 2014).

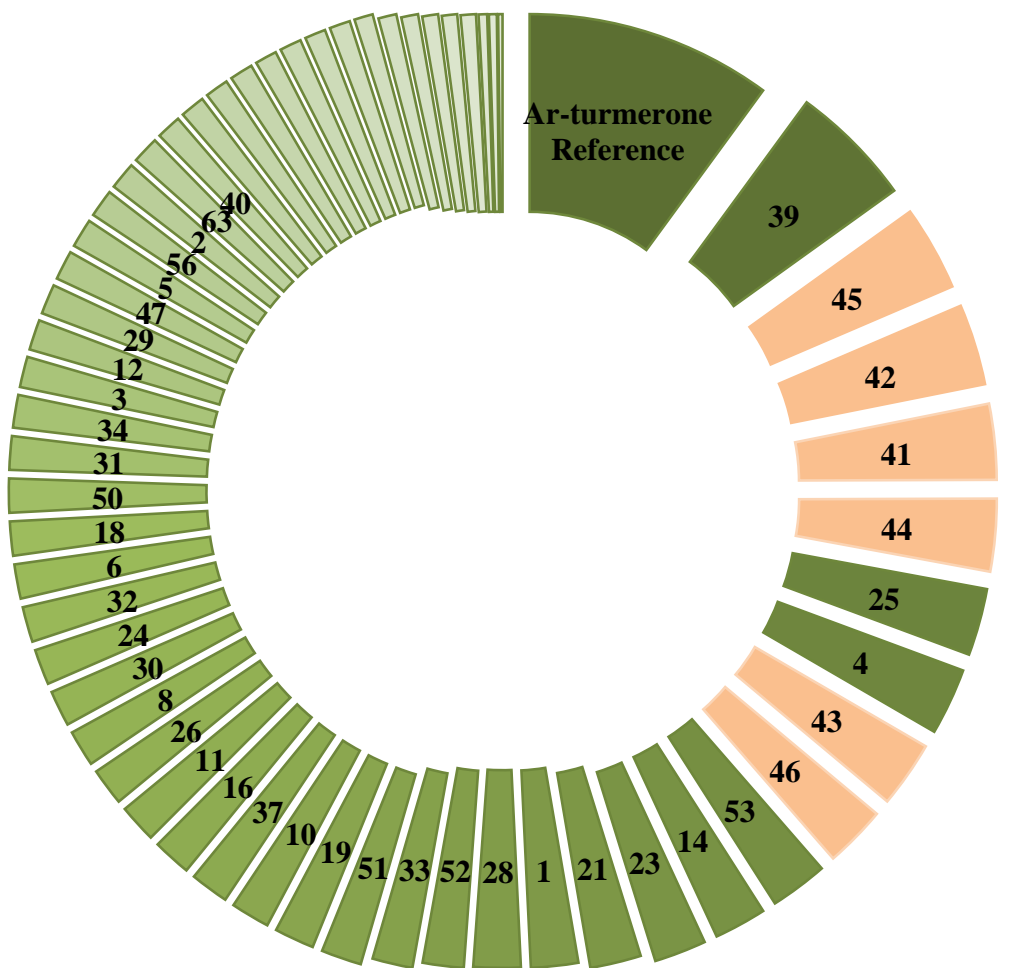

Figure 2: Chart showing turmeric finished products (S1-S56) in descending order, according to their average peak intensity in the chemical shift region related to Ar-turmerone peak (7.04-7.13 ppm). Pukka products (S41-46) are highlighted in pale orange (see Table 4). 


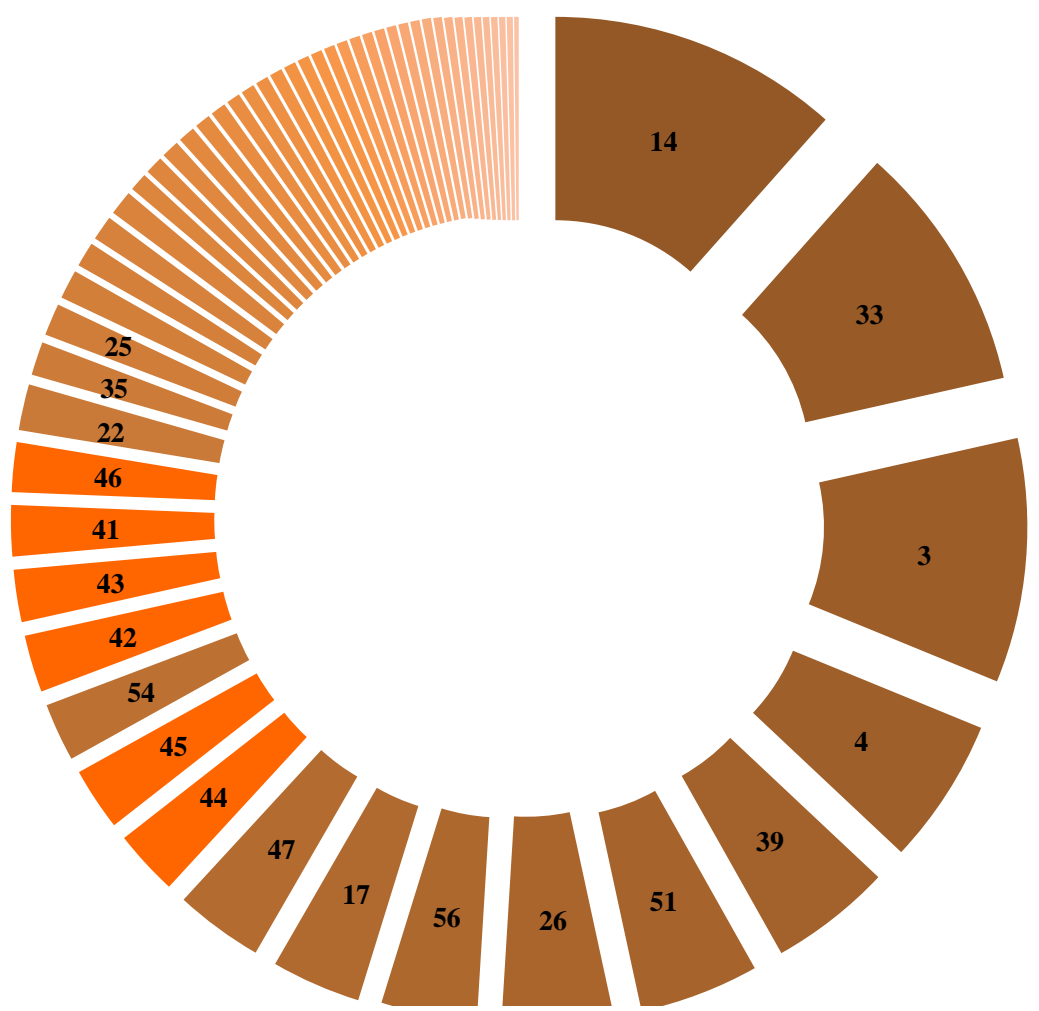

Figure 3: Chart showing turmeric finished products (S1-S56) in descending order, according to their average peak intensity in the chemical shift region related to curcumin peak (6.73-6.84 ppm). Pukka products (S41-46) are highlighted in bright orange (see also Table 2).

In Fig. 2, ar-turmerone reference presented the higher average absorbance, as expected., S41-46 presented high average absorption values in the region related to ar-turmerone. Therefore, in these products the quantity of ar-turmerone appeared to be comparatively high. Sample 39 showed the highest average peak intensity among all samples, and, therefore, concentration in ar-turmerone.

S41-46 appeared to contain an average quantity of curcumin (Fig. 3). Although samples 14, 3 and 33 were placed outside the scores plot (Fig. 1), they presented the highest amount of curcumin. Subsequent HPTLC analysis showed that samples 3 and 33 were not $C$. longa, as they were only composed of curcumin. However, sample 14 presented all bands related to curcuminoids.

\section{Products from along the Pukka value chain}

Figure 4 shows several products obtained from four different stages of the Pukka value chain. Some samples were crude materials from different suppliers within the Pukka value chain (S63-72), others were products obtained from two different manufacturing stages within the production, including processed turmeric powders (S60-62) and extracted materials (S57-59), and finally several of them were finished commercial products (S41-46). Sample 42 was analysed twice in order to assess the reproducibility of the measurements, thus two spots are presented in the scores plot (S421, S422). 


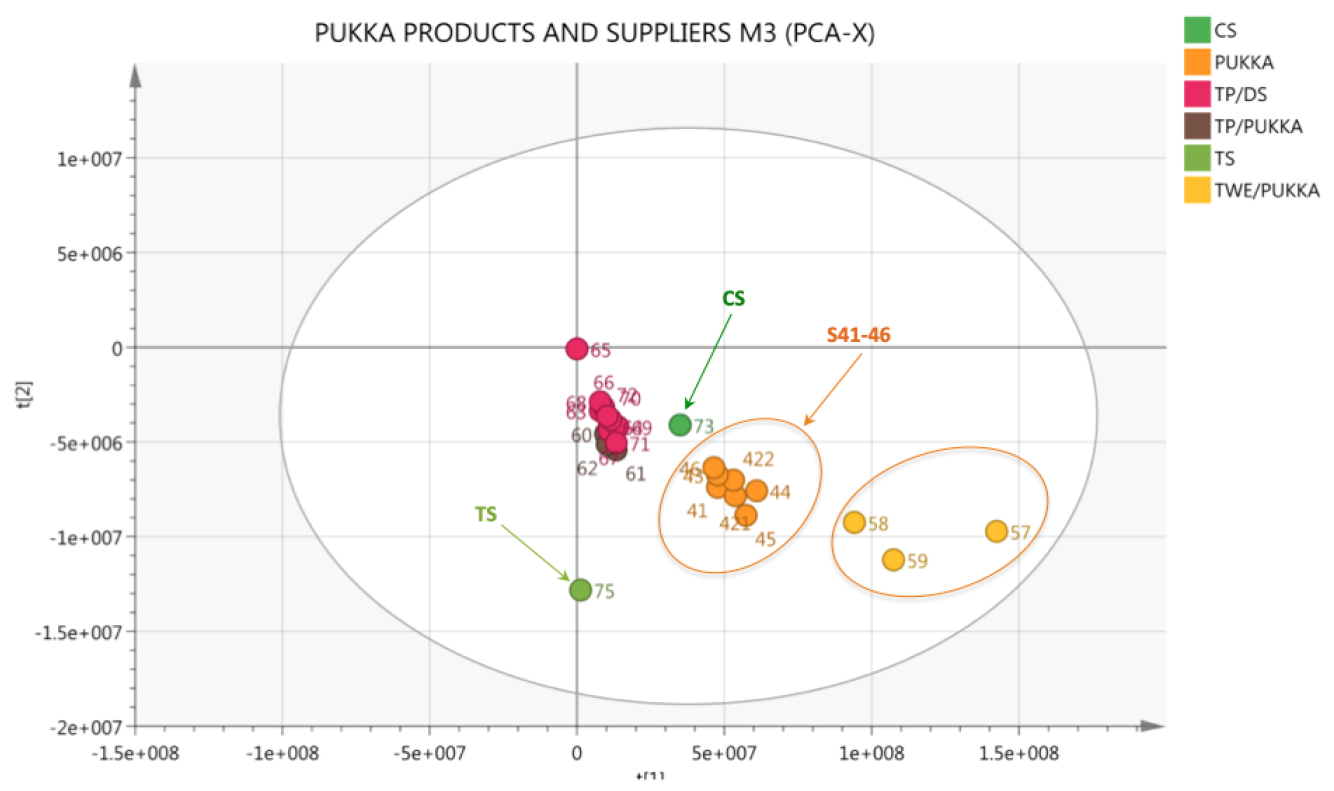

Figure 4: Scores plot in the chemical shift region 6-8 ppm, comparing products from four different stages of the Pukka value chain: finished products (PUKKA), extracted materials (TWE/PUKKA), processed powders (TP/PUKKA) and crude materials from different suppliers (TP/DS). Curcumin, piperine and ar-turmerone standards are also presented (CS, PS, TS). X axis: Principle Component 1 (PC1). Y axis: Principle Component 2 (PC2). $(n=25)$

Pukka finished products (S41-46, orange colour) appeared to be somewhere between Wholistic extracts and turmeric powders, as they are composed of a combination of these two preparations (Fig 4). This suggests that the quality of the final products is strongly influenced by the quality and concentration of the intermediate products obtained from the different manufacturing processes.

Extracts S57-59 were presented near the ar-turmerone standard, indicating that the extraction process used probably improves the concentration in active ingredients, and especially ar-turmerone (Fig 4). Pukka Turmeric powders (S60-62) were grouped with the crude powders obtained from different suppliers, showing similar metabolite profile to the starting materials.

As shown above, using NMR metabolomics, turmeric samples were successfully clustered into smaller groups based on their metabolite content. Samples containing turmeric powder were clearly separated from those containing turmeric extracts, indicating a variation in their chemical composition. In addition, the data obtained from NMR spectroscopy were effectively used for the quantitative analysis of the samples. However, this method was not able to provide information about why the clustering among turmeric products occurred. Furthermore, some samples containing botanicals other than $C$. longa, such as ginger (S39, 41-46) could not be differentiated from the rest of the samples. Finally, overlapping signals of different compounds or noise interference might also have occurred. Therefore, subsequent HPTLC analysis was performed to better understand the chemical composition of the samples. 


\section{HPTLC analysis}

72 turmeric samples both final products and crude materials were analysed using HPTLC. Two developing systems were used, one for the identification of curcuminoids and piperine and the other for the detection of essential oils. Curcumin, piperine and arturmerone standards were run together with the samples, in order to identify the presence of these compounds in the samples.

In Fig. 5, curcuminoids and piperine standards are illustrated on the top and all the finished turmeric products at the bottom $(\mathrm{S} 1-56)$. Curcumin is the top band $\left(\mathrm{R}_{\mathrm{F}}=0.39\right)$, demethoxycurcumin the middle band $\left(\mathrm{R}_{\mathrm{F}}=0.28\right)$ and bis-demethoxycurcumin the bottom band $\left(\mathrm{R}_{\mathrm{F}}=0.18\right)$. Piperine standard was illustrated as a yellow band at higher $\mathrm{R}_{\mathrm{F}}$ value than curcuminoids $\left(\mathrm{R}_{\mathrm{F}}=0.46\right)$.
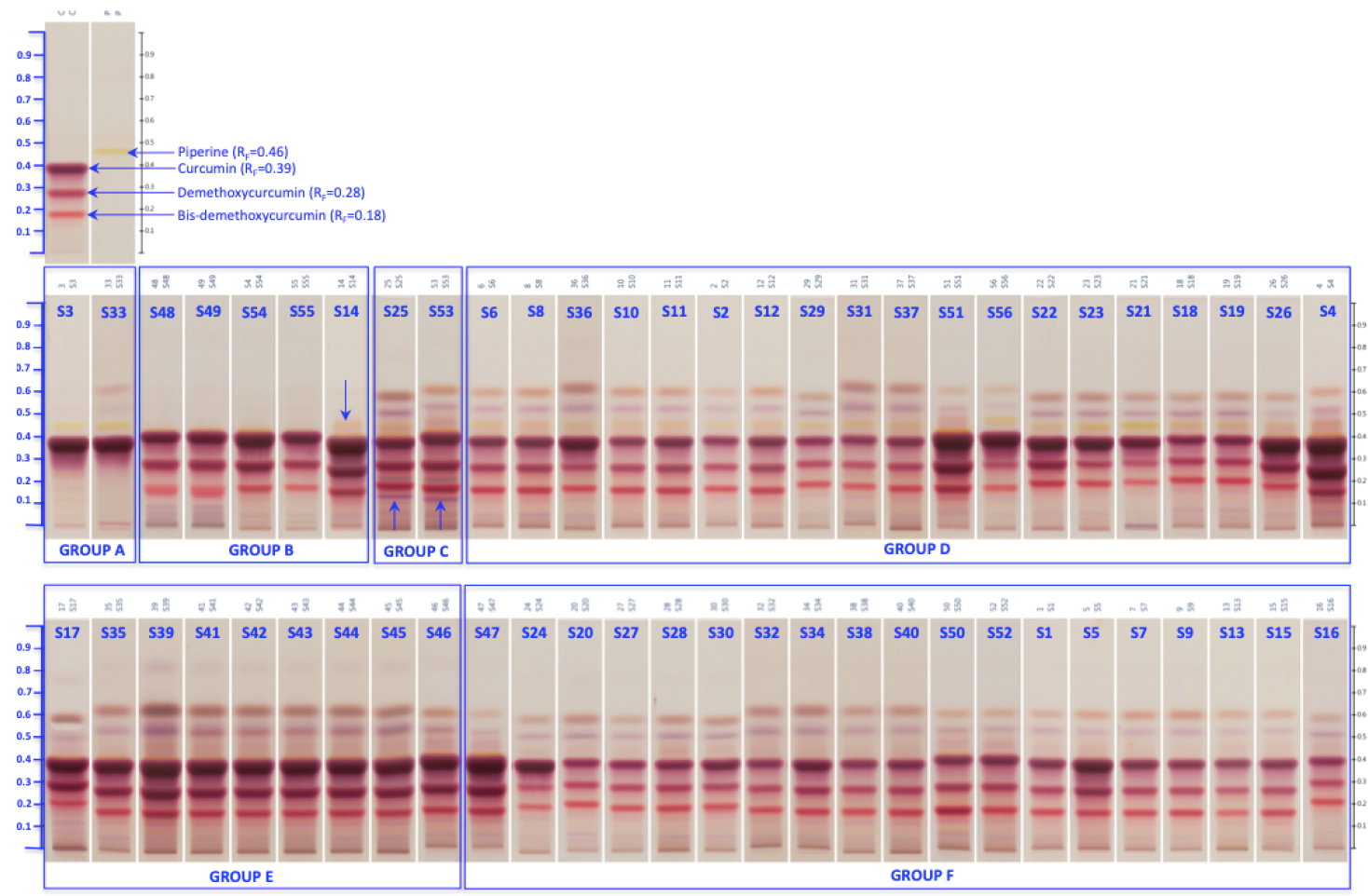

Figure 5: HPTLC plate under white light after derivatisation with anisaldehyde reagent, illustrating curcumin and piperine standards and samples 1-56, divided into groups (Group A: S3, 33, Group B: S48, 49, 54, 55, 14, Group C: S25, 53, Group D: S6, 8, 36, 10, 11, 2, 12, 29, 31, 37, 51, 56, 22, 23, 21, 18, 19, 26, 4, Group E: S17, 35, 39, 41-46, Group F: S47, 24, 20, 27, 28, 30, 32, 34, 38, 40, 50, 52, 1, 5, 7, 9, 13, 15, 16). Mobile phase toluene-acetic acid 4:1 (identification of curcuminoids).

Group A samples, extracts standardised to 95\% curcuminoids (S3, S33), showed two strong zones for curcumin and piperine $\left(\mathrm{R}_{\mathrm{F}}=0.39\right.$ and 0.46 respectively), but they did not present any of the other bands associated with the other curcuminoids. This indicates that these samples are not $C$. longa, confirming the results obtained from the PCA analysis (Fig. 1).

Group B extracts presented strong zones for all curcuminoids (Fig. 5). Among these samples, S14 was the only one showing a yellow band for piperine at $R_{F}=0.46$. The 
absence of any other bands higher than the characteristic piperine band is probably due to the fact that these samples did not contain crude powder, but only an extract standardised to $95 \%$ curcuminoids.

Group C samples had an intense purple band at $\mathrm{R}_{\mathrm{F}}=0.14$, and two other bands at $\mathrm{R}_{\mathrm{F}}=0.52$ and $\mathrm{R}_{\mathrm{F}}=0.61$. These bands were also found in the fingerprints of the crude powders from different suppliers (see Appendix 4). Thus, this indicates that these samples were composed of crude turmeric rhizomes.

Group D samples exhibited the three characteristic zones for curcuminoids, a strong zone for piperine and two other zones, found also in $C$. long $a$ crude powders, at $\mathrm{R}_{\mathrm{F}}=0.52$ and $\mathrm{R}_{\mathrm{F}}=0.61$. As expected, $\mathrm{S} 21$ presented the strongest piperine band. Samples 51, 22, 26 and 4 showed intense bands for all curcuminoids.

Group E samples had similar bands to Group D, but they also presented a faint extra band at $\mathrm{R}_{\mathrm{F}}=0.81$. According to the HPTLC association $C$. longa samples should not show a purple zone at $\mathrm{R}_{\mathrm{F}}=0.81$ and $\mathrm{R}_{\mathrm{F}}=0.57$, as this could indicate adulteration with other Curcuma species. Sample 39 presented the strongest zone for this $\mathrm{R}_{\mathrm{F}}$ value $\left(\mathrm{R}_{\mathrm{F}}=0.81\right)$. This could have happened because $\mathrm{S} 39$ also contained ginger or due to adulteration with other Curcuma species. The yellow zone for piperine could not be clearly identified in samples S41-46, as their fingerprint at $\mathrm{R}_{\mathrm{F}}=0.4-0.5$ was blurry. Sample 17 claimed to contain the Longvida ${ }^{\circledR}$ optimized extract. However, the extra zones present indicate that it may be adulterated with other species. Also, the other bands related to $C$. longa significantly differed from the rest of the samples at the $\mathrm{R}_{\mathrm{F}}$ values.

Group F samples showed all bands known to represent curcuminoids, with samples 5 and 47 presenting the strongest zones. Some of these samples claimed to contain piperine (S 5, 7, 9, 13, 20, 24, 28, 30, 34, 38), while others did not contain any piperine, such as samples 47, 1, 15, 16, 27, 32, 40, 50 and 52. Although some samples did not contain any piperine, they showed a pale pink band at $R_{F}=0.46$ (piperine zone).

In Fig. 6, the ar-turmerone standard is illustrated on the top $\left(\mathrm{R}_{\mathrm{F}}=0.59\right)$. Group A extracts appeared to contain no or minimum amounts of ar-turmerone and other turmeric essential oils. This may have happened due to age, as the potency of volatile oils is lost over time (Turek and Stintzing, 2013). Specifically, sample 3 showed only two zones at very low $\mathrm{R}_{\mathrm{F}}$ values, confirming that it was not $C$. longa (see also Fig. 5) 

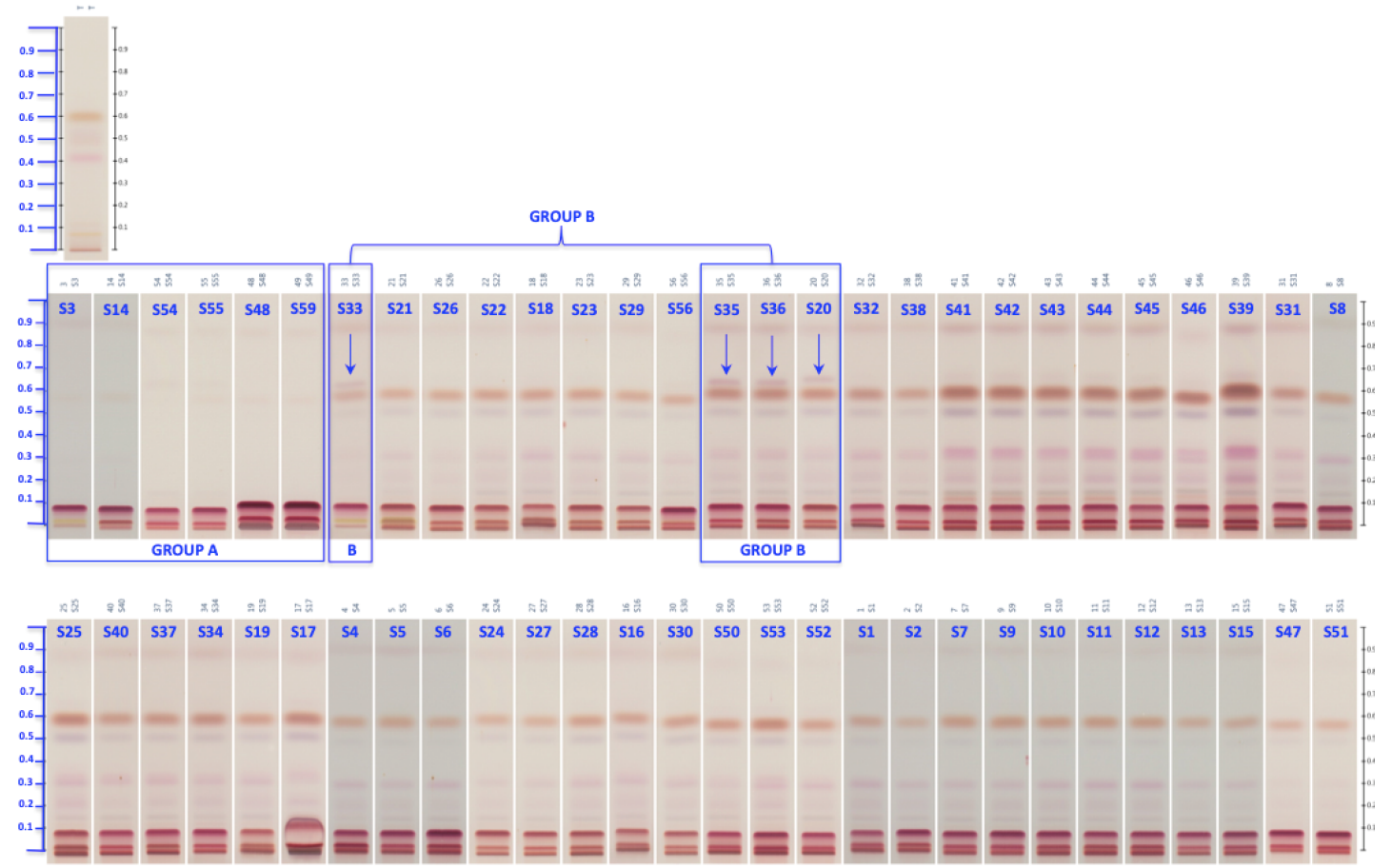

Figure 6: HPTLC plate under white light after derivatisation using anisaldehyde reagent, illustrating Ar-turmerone standard and samples 1-56, divided into groups (Group A: S3, 14, 54, 55, 48, 59, Group B: S33, 35, 36, 20). Mobile phase dichloromethane (identification of essential oils).

Although, Group B samples contained ar-turmerone, they differed from the other samples, as they exhibited an extra zone at $\mathrm{R}_{\mathrm{F}}=0.63$. These samples also presented a faint zone at $\mathrm{R}_{\mathrm{F}}=0.89$, which was also present in the fresh rhizomes, according to Booker (2014). As confirmed by the HPTLC analysis of curcuminoids, sample 33 was not C. longa (Fig. 5).

S41-46 and sample 39 (Group C) presented the strongest zones for ar-turmerone and a very strong zone at $\mathrm{R}_{\mathrm{F}}=0.89$, possibly because they were obtained from a shorter supply chain, and, therefore, they presumably were fresher. These samples also showed some extra bands at intermediate $\mathrm{R}_{\mathrm{F}}$ values (e.g. at $\mathrm{R}_{\mathrm{F}}=0.1$ ), probably due to the presence of other ingredients, such as ginger.

All the other samples contained ar-turmerone and presented similar fingerprints. Their only difference was based on the band at $\mathrm{R}_{\mathrm{F}}=0.89$. Some samples did not produce this band, such as samples $23,56,52,53,50,47$ and 51 or presented a very pale zone (S21, 26, 22, 18, 25 etc.), probably because they were older (Fig. 6).

In Figure 7, Pukka finished (S41-46) and intermediate products (S57-62), as well as starting materials from different suppliers (S63-72) are illustrated. Curcuminoids standard (C) is also presented, with curcumin being the top band $\left(\mathrm{R}_{\mathrm{F}}=0.39\right)$, demethoxycurcumin the middle band $\left(\mathrm{R}_{\mathrm{F}}=0.28\right)$ and bis-demethoxycurcumin the bottom band $\left(\mathrm{R}_{\mathrm{F}}=0.18\right)$. 


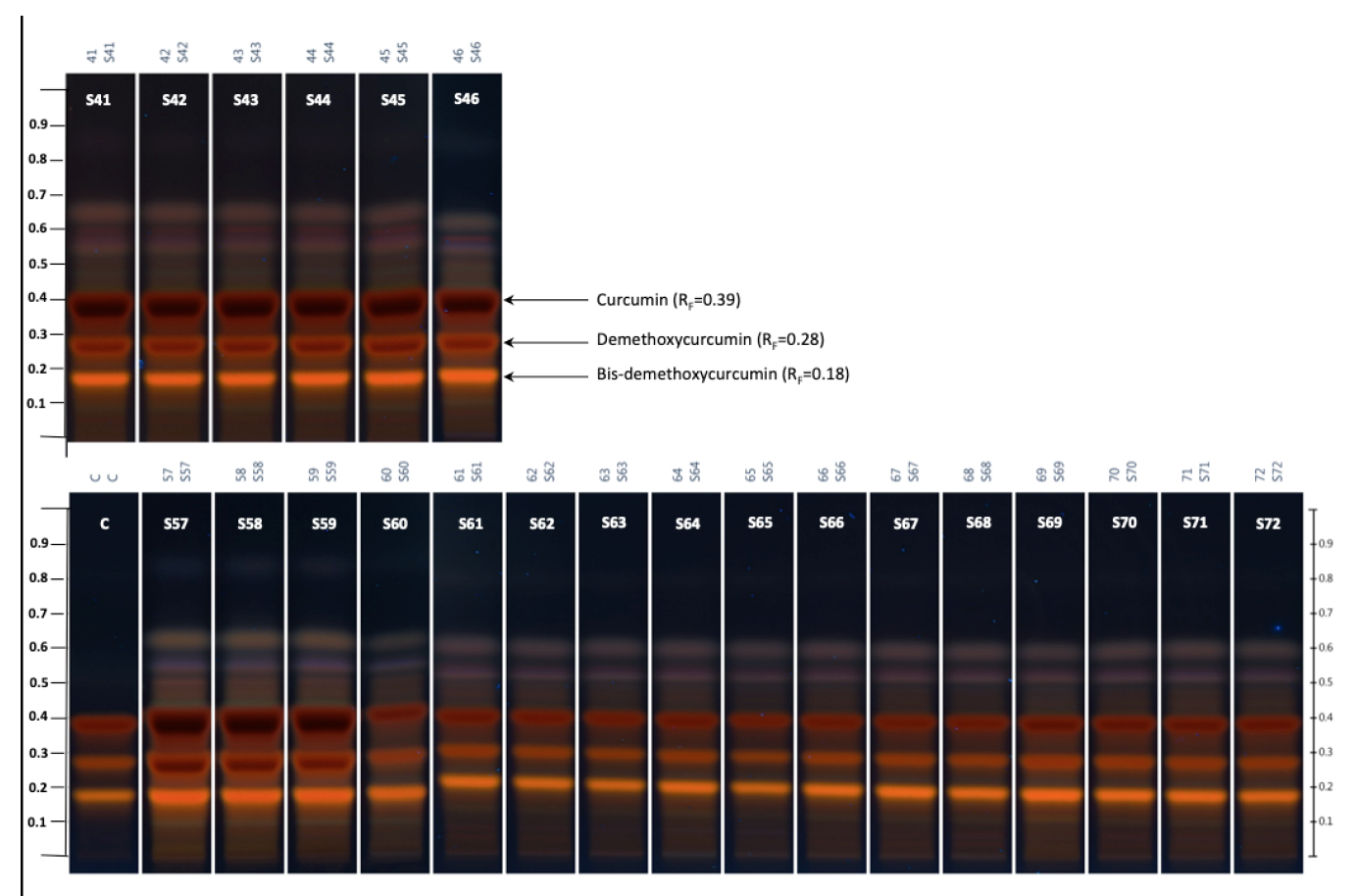

Figure 7: HPTLC plate under UV light $(366 \mathrm{~nm})$ after derivatisation with anisaldehyde reagent, illustrating Pukka finished (S41-46) and intermediate products (S57-62), curcumin standard (C) and crude turmeric powders (S63-72). Mobile phase toluene-acetic acid 4:1 (identification of curcuminoids).

All crude powders from different suppliers (S63-72) are C. longa, as they present all the bands related to curcuminoids. In addition, they showed two extra zones at higher $\mathrm{R}_{\mathrm{F}}$ values $\left(\mathrm{R}_{\mathrm{F}}=0.52\right.$ and $\left.\mathrm{R}_{\mathrm{F}}=0.61\right)$, which were also presented in almost all finished turmeric products (see Fig. 5). Sample 65 showed the weakest zones for curcuminoids, while samples 69 and 71 the strongest ones among the crude materials. Pukka turmeric powders (S60-62) exhibited the same bands as crude turmeric powders and almost the same concentration. S57-59 on the other hand, showed the strongest zones for curcuminoids, indicating that the extraction process improves the concentration of key compounds.

All samples showed the characteristic band related to ar-turmerone at $\mathrm{R}_{\mathrm{F}}=0.59$ (Figure 8). S57-59 (Wholistic Extracts) presented the strongest bands among all samples. Finished products S41-46 also displayed a strong zone for ar-turmerone, stronger than Pukka turmeric powders (S60-62). This is because the final products were prepared using a combination of powder and extract.

HPTLC is a low-cost and flexible technique that enables the detailed investigation of the chemical composition of multiple samples. As described above, using this method the compounds presented in turmeric samples were easily separated and observed visually. There were samples that did not contain all active ingredients of $C$. longa, illustrated by incomplete fingerprints, and others that contained additional ingredients or botanicals, and thus exhibited more complex fingerprints than expected. However, this method also involves some limitations, such as the low sensitivity and separation power. This explains the fact that several turmeric samples exhibited a band at $R_{F}=0.46$, although they did not contain piperine as an ingredient. Therefore, the combination of 
the two analytical techniques, NMR metabolomics and HPTLC, was necessary to obtain valid and consistent results.

\section{Conclusions}

In this study ${ }^{1} \mathrm{H}-\mathrm{NMR}$ metabolomics and HPTLC analytical techniques were applied to investigate the quality of commercial turmeric products available in the UK, as well as the chemical variation of turmeric products obtained from specific stages within the Pukka value chain, including starting materials, processed powders, extracted materials and end products. Both methods exhibited interesting, complementary data and it was possible to provide a qualitative and quantitative analysis of turmeric samples. Curcuminoids and turmeric essential oil components were the main medicinally important compounds analysed. The presence of piperine was also investigated within the samples, as this compound has been widely used in turmeric preparations, promoted for improving the bioavailability of curcumin.

Regarding the commercial products, the results showed that there was an important variation in the chemical composition of turmeric products, ranging in concentration and number of active ingredients. The products marketed as containing only turmeric powder presented a similar metabolite profile. However, important chemical variation appeared in the products composed of turmeric extracts or a combination of extracts and powder. Some extracts were found to contain only curcumin, while others did not contain any essential oils but did have the three basic curcuminoids. There were also several samples that contained ingredients additional to those from $C$. longa, such as ginger, which were expected according to their formulation claims. The variation among turmeric extracts indicates that the manufacturing practices, including the extraction process of the crude material, play a major role in the final product quality, requiring an integrated approach along the entire value chain of a product. S41-46 were shown to be among the turmeric products with the highest concentration of curcuminoids and particularly the essential oil components.

Some samples appeared to contain significant amounts of piperine, while others with such a label claim either had very small quantities, almost undetectable levels or no piperine. There were also samples that, although they did not contain any piperine as an ingredient, exhibited a pink band at $\mathrm{R}_{\mathrm{F}}=0.46$.

Turmeric products obtained from various stages of the Pukka value chain, presented similar metabolite variability and quality, typical for an integrated quality control system. All crude powders from different suppliers were $C$. longa. Processed powders appeared to have identical chemical composition to the crude biological materials. In addition, intermediate products S57-S59 exhibited significantly higher concentration in curcuminoids and ar-turmerone than the powders, indicating that the extraction process applied leads to enhanced quality extracts, and, therefore, improves end product quality.

Until there is consensus about which constituents and additives are most important to harness the anti-inflammatory benefits of turmeric, the composition of different products will continue to vary widely. The great variety in products leads to difficulty in comparing the different products available and in interpreting clinical trials and other 
research into the effects of turmeric. Future research is needed to ascertain the relative importance of different constituents and any synergy that may happen between them.

This study has focused on C. longa, which is one of the most popular species within the genus of Curcuma. Since its popularity continuously increases and these products become more accessible to consumers, it is very important that commercial products are of acceptable quality and safety.

Overall, this study shows that high value-added plant-based products can be produced using carefully controlled processing steps throughout the value chain. Specific EU standards and more stringent regulations need to be designed and implemented for dietary supplements, to further guarantee the products' quality and safety.

\section{Acknowledgements}

Loukiani Chatzinasiou was funded through a charitable donation from Pukka Herbs Ltd. UK. Anthony Booker was part funded through a donation from CONICyT, Chile.

\section{Conflict of Interest Statement}

Loukiani Chatzinasiou was part funded by Pukka herbs Ltd. for this project.

Pukka herbs Ltd. provided funding for this research project but did not take part in the experimental stages or in the data interpretation.

\section{References}

Aggarwal, B.B., Yuan, W., Li, S., Gupta, S.C., 2013. Review: Curcumin $\square$ free turmeric exhibits anti $\square$ inflammatory and anticancer activities: Identification of novel components of turmeric. Molecular Nutrition \& Food Research 57(9), 1529-1542.

Ammon, H.P.T., Anazodo, M.I., Safayhi, H., Dhawan, B.N., Srimal, R.C., 1992. Curcumin: A Potent Inhibitor of Leukotriene B4 Formation in Rat Peritoneal Polymorphonuclear Neutrophils (PMNL). Letters 58, 226.

Anand, P., Kunnumakkara, A.B., Newman, R.A., Aggarwal, B.B., 2009. Bioavailability of Curcumin: Problems and Promises. Molecular Pharmaceutics 4(6), 807-818.

Booker, A., 2014. The Transformation of Traditional Asian Medical Knowledge into International Commodities - the Link between Traditional Medicines and the International Market. pp. 1-448.

Booker, A., Frommenwiler, D., Johnston, D., Umealajekwu, C., Reich, E., Heinrich, M., 2014a. Chemical variability along the value chains of turmeric (Curcuma longa): 
A comparison of nuclear magnetic resonance spectroscopy and high performance thin layer chromatography. Journal of Ethnopharmacology 152, 292-301.

Booker, A., Frommenwiler, D., Johnston, D., Umealajekwu, C., Reich, E., Heinrich, M., 2014b. Chemical variability along the value chains of turmeric (Curcuma longa): A comparison of Nuclear Magnetic Resonance Spectroscopy and High Performance Thin Layer Chromatography. Journal of Ethnopharmacology doi: 10.1016/j.jep.2013.12.042.

Dixit, S., Purshottam, S.K., Khanna, S.K., Das, M., 2009. Surveillance of the quality of turmeric powders from city markets of India on the basis of curcumin content and the presence of extraneous colours. Food Additives \& Contaminants: Part A 26(9), 12271231.

Kukula-Koch, W., Grabarska, A., Luszczki, J., Stepulak, A., 2018. Superior anticancer activity is demonstrated by total extract of Curcuma longa L. as opposed to individual curcuminoids separated by centrifugal partition chromatography. Phytotherapy Research 32(6), 1-10.

Li, S., Yuan, W., Guangrui, D., Wang, P., Yang, P., Aggrawal, B.B., 2011. Chemical composition and product quality control of turmeric (Curcuma longa L.). Pharmaceutical Crops 1, 28-54.

Maharao, N.V., Joshi, A.A., Gerk, P.M., 2016. Special Issue: Inhibition of glucuronidation and oxidative metabolism of buprenorphine using GRAS compounds or dietary constituents/supplements: in vitro proof of concept. Biopharmaceutics \& Drug Disposition 38(2), 87-93.

Park, S.Y., Jin, M.L., Kim, Y.H., Kim, Y.H., Lee, S.J., 2012. Anti-inflammatory effects of aromatic-turmerone through blocking of NF- $\mathrm{BB}, \mathrm{JNK}$, and p38 MAPK signaling pathways in amyloid $\beta$-stimulated microglia. International immunopharmacology 14(1), 13-20.

Prasad, S., Aggarwal, B.B., 2011. Chapter 13: Turmeric, the Golden Spice, in: Benzie, I.F.F., Wachtel-Galor, S. (Eds.), Herbal Medicine: Biomolecular and Clinical Aspects. CRC Press/ Taylor \& Francis.

Prasad, S., Tyagi, A.K., Aggarwal, B.B., 2014. Recent Developments in Delivery, Bioavailability, Absorption and Metabolism of Curcumin: the Golden Pigment from Golden Spice. Cancer Res Treat. 46(1), 2-18.

Ravindranath, V., Chanfrasekhara, N., 1981. IN VITRO STUDIES ON THE INTESTINAL ABSORPTION OF CURCUMIN IN RATS. Toxicology 20, 251-257.

Santosh, S.K., Panday, M.K., Sung, B., Ahn, K.S., Murakami, A., Sethi, G., Limtrakul, P., Badmaev, V., Aggarwal, B.B., 2017. Curcumin, demethoxycurcumin, bisdemethoxycurcumin, tetrahydrocurcumin and turmerones differentially regulate anti-inflammatory and anti-proliferative responses through a ROS-independent mechanism. Carcinogenesis 28(8), 1765-1773.

Shishu, M.M., 2010. Journal of Functional foods 2, 60-65.

Starling, S., 2009. Tainted turmeric supplements linked to Scandanavian deaths. $<$ http://www.nutraingredients.com/Regulation/Tainted-turmeric-supplements-linkedto-Scandinavian-deaths $>$ (accessed 8-10-2013.2013).

Turek , C., Stintzing, F.C., 2013. Stability of Essential Oils: A Review. Comprehensive Reviews in Food Science and Food Safety 12, 40-53.

Wang, Y.-J., Pan, M.-H., Cheng, A.-L., Lin, L.-I., Ho, Y.-S., Hsieh, C.-Y., Lin, J.-K., 1997. Stability of curcumin in buffer solutions and characterization of its degradation products. Journal of Pharmaceutical and Biomedical Analysis 15, 1867-1876. 
